\title{
The Boltzmann-Hamel equations for the optimal control of mechanical systems with nonholonomic constraints
}

\author{
Jared M. Maruskin ${ }^{1, *, \dagger}$ and Anthony M. Bloch ${ }^{2}$ \\ ${ }^{1}$ Department of Mathematics, The San José State University, San José, CA 95192-0103, U.S.A. \\ ${ }^{2}$ Department of Mathematics, University of Michigan, Ann Arbor, MI, U.S.A.
}

\begin{abstract}
SUMMARY
In this paper, we generalize the Boltzmann-Hamel equations for nonholonomic mechanics to a form suited for the kinematic or dynamic optimal control of mechanical systems subject to nonholonomic constraints. In solving these equations one is able to eliminate the controls and compute the optimal trajectory from a set of coupled first-order differential equations with boundary values. By using an appropriate choice of quasi-velocities, one is able to reduce the required number of differential equations by $m$ and $3 m$ for the kinematic and dynamic optimal control problems, respectively, where $m$ is the number of nonholonomic constraints. In particular we derive a set of differential equations that yields the optimal reorientation path of a free rigid body. In the special case of a sphere, we show that the optimal trajectory coincides with the cubic splines on $S O(3)$. Copyright (C) 2010 John Wiley \& Sons, Ltd.
\end{abstract}

Received 5 December 2008; Revised 11 March 2010; Accepted 17 March 2010

KEY WORDS: nonholonomic constraints; optimal control; variational principles; Boltzmann-Hamel equations; quasi-velocities

\section{INTRODUCTION}

In this paper, we extend the classical Boltzmann-Hamel equations to kinematic and dynamic optimal control problems, the latter formalism being a fourth-order generalization of these equations. In the analysis of nonholonomic systems, a number of different formalisms have emerged based on a set of quantities known as quasi-velocities, see for example [1-3]; and, for a more geometric treatment, [4,5]. For an $n$ degree of freedom system with $m<n$ nonholonomic constraints, one defines $m$ quasi-velocities $u^{\sigma}, \sigma=n-m+1, \ldots, n$, in such a way that they span the constraint distribution. In this way the constraints reduce to the relations $u^{\sigma}=0$ and one only need to solve for the remaining $n-m$ independent quasi-velocities. In addition, the $n$ kinematic relations which define the constraints can be numerically integrated to produce the curve of motion. One requires in total $2 n-m$ differential equations of motion, as opposed to the $2 n+m$ equations initially necessary using the Lagrange-D'Alembert equations $[1,6]$. In practice, however, one must often differentiate the constraint equations, yielding a coupled system of $n+m$ second-order differential equations. Numerical instabilities often arise when numerically integrating these equations of motion [1].

\footnotetext{
${ }^{*}$ Correspondence to: Jared M. Maruskin, Department of Mathematics, The San José State University, San José, CA 95192-0103, U.S.A.

†E-mail: maruskin@math.sjsu.edu
}

Contract/grant sponsor: Publishing Art Research Council; contract/grant number: 98-1846389

Contract/grant sponsor: NSF; contract/grant numbers: DMS-0604307, DMS-0907949, CMS-0408542 
These numerical instabilities do not exist when utilizing quasi-velocity techniques by virtue of the fact that the coordinates choosen for the velocities automatically satisfy the constraints by virtue of their geometry. In addition to there being fewer equations of motion, if one makes a judicious choice of quasi-velocities, these equations of motion are oftentimes simpler than the equations of motion that arise using the fundamental nonholonomic form of Lagrange's equations. In nonholonomic mechanics, there is no set procedure for choosing the free quasi-velocities; normally they are chosen to be as simple as possible. In extending these techniques to kinematic and dynamic optimal control problems, however, a full set of quasi-velocities will be prescribed based on the control vector fields, as we shall discuss in this paper.

Given a mechanical system with nonholonomic constraints, one may reexpress the Lagrangian in terms of the generalized coordinates and the quasi-velocities. This new Lagrangian will not, in general, satisfy the Euler-Lagrange equations. Instead it satisfies a similar set of equations known as the Boltzmann-Hamel equations.

The Boltzmann-Hamel equations have also been used for the analysis of unconstrained systems with symmetry. For instance, in rigid body mechanics the body-axis components of the angular velocity constitute a set of quasi-velocities. The Boltzmann-Hamel equations for this set of quasivelocities produce Euler's equations for rigid body dynamics [1]. As a generalization of Euler's equations, the Euler-Poincaré equations are a set of dynamical equations of motion for mechanical systems with symmetry, i.e. systems whose underlying configuration manifold is a Lie group [7, 8]. It was shown in [9] that the Euler-Poincare equations are a special case of the Boltzmann-Hamel equations, under the conditions that (i) the underlying configuration manifold is a Lie group, (ii) the quasi-velocities are taken to be the pullback of the velocity vector to the Lie algebra by the left translation map, and (iii) the Lagrangian is left-invariant. The Euler-Poincaré equations have been generalized to a set of Euler-Poincaré optimal control equations, see for instance [10-12]. In this way, the Boltzmann-Hamel optimal control equations presented here can be thought of as a generalization of the Euler-Poincaré optimal control equations to systems in which the Lagrangian is not left-invariant or to systems without symmetry.

In the optimal control problem one has a cost function that one seeks to minimize, usually taken to be a line integral over the curve of motion. In kinematic or dynamic optimal control, the path itself is subject to a certain set of kinematic or dynamic equations of motion, respectively. If one uses Pontryagin's Maximum Principle, each of these equations is enforced by a corresponding Lagrange multiplier [13-15]. The formalism we present here has the additional feature that it circumvents the necessity of this set of Lagrange multipliers. $m$ Lagrange multipliers are still required to enforce the nonholonomic constraints, an inescapable feature of the vakonomic problem, e.g. see $[1-3,5,14,16,17]$. In the kinematic optimal control problem, the set of $n$ differential equations for the Lagrange multipliers used to enforce the kinematic equations of motion are replaced with $n-m$ differential equations for the quasi-velocities. In the dynamic optimal control problem, the set of differential equations for the $2 n$ Lagrange multipliers used to enforce the dynamical equations of motion are replaced with $2 n-2 m$ differential equations for the independent quasi-accelerations and quasi-jerks; the $n$ differential equations for the velocities are further replaced by $n-m$ differential equations for the quasi-velocities.

As a final example we consider the optimal control of the free rigid body, given a set of control torques placed along the axes of the body-fixed frame. When using the body-axis components of the angular velocity as quasi-velocities, the classical Boltzmann-Hamel equations for nonholonomic mechanics produce Euler's equations for rigid body dynamics. By applying the dynamical optimal control Boltzmann-Hamel equations to this set of quasi-velocities, we derive a fourth-order version of Euler's equations whose solution coincides with the optimal reorientation problem. The problem of finding the optimal reorientation of a free rigid body gained some publicity in recent years; as NASA used pseudospectral optimal control theory to reorient the International Space Station using onboard control moment gyros, see [18-21]. As a special case of the fourth-order Euler equations, we consider the optimal reorientation of a free rigid sphere, showing that the equations defining the optimal trajectory coincide with the differential equations that define cubic splines on $S O(3)$, a set of equations first derived in [22]. 
Table I. Summation convention.

\begin{tabular}{llll}
\hline Letter type & Indices & Summation over & \multicolumn{1}{c}{ Summation over } \\
\hline Capital & $I, J, K$ & $1, \ldots, n-m$ & Unconstrained dimensions \\
Greek & $\sigma, \tau$ & $n-m+1, \ldots, n$ & Constrained dimensions \\
Lower case & $a, b, i, j, k$ & $1, \ldots, n$ & All dimensions \\
\hline
\end{tabular}

A preliminary version of this paper was published in The Conference Proceedings of the 46th IEEE Conference on Decision and Control [23].

\subsection{Summation convention}

To aid in notation, we will invoke the summation convention throughout this paper for an $n$ degree of freedom system with $m<n$ nonholonomic constraints. The capital letters $I, J$, and $K$ run over the unconstrained dimensions $1, \ldots, n-m$. The Greek letters $\sigma$ and $\tau$ run over the constrained dimensions $n-m+1, \ldots, n$. Finally, the lower case letters $a, b, i, j$, and $k$ run over all dimensions $1, \ldots, n$ (Table I).

\section{QUASI-VELOCITIES AND VARIATIONS}

In this section, we will present the basic background on nonholonomic constraints and quasivelocities. We will discuss the basic properties of this connection and derive the transpositional relations [1-3]. We will follow the notation for the geometric formalism laid out in [5].

\subsection{Nonholonomic constraints and quasi-velocities}

Let $Q$ be the $n$-dimensional, smooth configuration manifold of our system, $L: T Q \rightarrow \mathbb{R}$ a Lagrangian for the system, where the $2 n$-dimensional manifold $T Q$ is the tangent bundle of $Q$, and $\mathcal{D}$ is a smooth, $(n-m)$-dimensional, nonintegrable distribution, which may be described as the null space of a set of constraint one-forms $\omega^{\sigma}$ as follows:

$$
\mathscr{D}=\left\{(q, v) \in T Q: \omega^{\sigma}(v)=0\right\} .
$$

The Lagrangian is typically given by the kinetic minus potential energy of the system. A velocity vector $\dot{q}$ at $q$ is said to be kinematically admissible if $\dot{q} \in \mathscr{D}_{q} \subset T_{q} Q$. In local coordinates, the constraint one-forms are expressed as $\omega^{\sigma}=a_{i}^{\sigma} d q^{i}$, so that the velocity of a curve in the distribution $\mathscr{D}$ satisfies the nonholonomic constraints

$$
a_{i}^{\sigma}(q) \dot{q}^{i}=0
$$

for $\sigma=n-m+1, \ldots, n$. Given a manifold with a constraint distribution, one defines the quasivelocities as follows.

\section{Definition 1}

Given a closed curve $c:\left[t_{1}, t_{2}\right] \rightarrow Q$ and an open set $U \subset Q$ containing $c$, define the vector fields $\mathscr{E}=\left\{E_{1}, \ldots, E_{n}\right\}$ such that, at each $q \in U$, the vectors $E_{1}, \ldots, E_{n} \in T_{q} Q$ are linearly independent and thus form a basis of $T_{q} Q$. Then the quasi-velocities of the curve $c$ with respect to the basis $\mathscr{E}$ are the $n$ scalar functions $u^{i}:\left[t_{1}, t_{2}\right] \rightarrow \mathbb{R}$ defined such that $\dot{c}(t)=u^{i}(t) E_{i}(c(t))$, i.e. they are the components of the velocity $\dot{c}$ with respect to the moving frame $\mathscr{E}$.

Let $E^{1}, \ldots, E^{n} \in T_{q}^{*} Q$ be the basis dual to the vectors $E_{1}, \ldots, E_{n} \in T_{q} Q$ at $q$. In practice, the vector fields $\mathscr{E}$ are chosen so that, at each $q \in U$, the dual one-forms $E^{1}, \ldots, E^{m} \in T_{q}^{*} Q$ coincide with the constraint one-forms; i.e., $E^{\sigma}=\omega^{\sigma}$ for $\sigma=n-m+1, \ldots, n$.

Let $\Psi_{j}^{i}$ be the matrix whose rows are the one-forms $E^{1}, \ldots, E^{n}$ and let $\Phi_{j}^{i}$ be the matrix whose columns are the vectors $E_{1}, \ldots, E_{n}$; i.e. $E^{i}=\Psi_{j}^{i} d q^{j}$ and $E_{j}=\Phi_{j}^{i} \partial / \partial q^{i}$. The matrices $\Psi_{j}^{i}$ and $\Phi_{j}^{i}$ 
are therefore inverses of each other. One uses these matrices to convert back and forth from the coordinate to quasi-velocities via the relations

$$
u^{i}=\Psi_{j}^{i} \dot{q}^{j} \quad \text { and } \quad \dot{q}^{i}=\Phi_{j}^{i} u^{j}
$$

Notice that, by construction, the last $m$ rows of the transformation matrix $\Psi_{j}^{i}$ must coincide with the constraint matrix, i.e. $\Psi_{i}^{\sigma}(q)=a_{i}^{\sigma}(q)$. Therefore, in terms of the quasi-velocities, the nonholonomic constraints are $u^{\sigma}=0$, for $\sigma=n-m+1, \ldots, n$.

Given a curve $c:\left[t_{1}, t_{2}\right] \rightarrow Q$ with local coordinates $q^{i}(t)$, the velocity $\dot{c}:\left[t_{1}, t_{2}\right] \rightarrow T Q$ can be represented equivalently in any of the following ways:

$$
\dot{c}=\dot{q}^{i} \frac{\partial}{\partial q^{i}}=\dot{q}^{j} \Psi_{j}^{k} \Phi_{k}^{i} \frac{\partial}{\partial q^{i}}=u^{k} E_{k} .
$$

\subsection{Variations}

\section{Definition 2}

Consider a curve $c:\left[t_{1}, t_{2}\right] \rightarrow Q$. A variation of $c$ is a smooth function $\vartheta:[-\varepsilon, \varepsilon] \times\left[t_{1}, t_{2}\right] \rightarrow Q$ that satisfies the following conditions:

(i) $\vartheta(0, t)=c(t), \forall t \in\left[t_{1}, t_{2}\right]$

(ii) $\vartheta\left(s, t_{1}\right)=c\left(t_{1}\right)$ and $\vartheta\left(s, t_{2}\right)=c\left(t_{2}\right), \forall s \in[-\varepsilon, \varepsilon]$.

\section{Definition 3}

The infinitesimal variation $\delta c:\left[t_{1}, t_{2}\right] \rightarrow T Q$ corresponding to the variation $\vartheta$ is the vector field defined along $c$ by $\delta c(t)=\left.\frac{\partial \vartheta(s, t)}{\partial s}\right|_{s=0}$.

We will denote the components of $\delta c$ with respect to the coordinate basis $\left\{\partial / \partial q^{i}\right\}$ as $\delta q^{i}$, so that $\delta c=\delta q^{i} \partial / \partial q^{i}$. With respect to the basis $\mathscr{E}$ we have.

\section{Definition 4}

The quasi-infinitesimal variations $\zeta^{i}$ with respect to the basis $\mathscr{E}$ are a set of $n$ scalar functions $\zeta^{i}:\left[t_{1}, t_{2}\right] \rightarrow \mathbb{R}$ defined such that $\delta c(t)=\zeta^{i}(t) E_{i}(c(t))$.

Notice that to compute the quasi-infinitesimal variations one can use the transformation relations $\zeta^{i}(t)=\Psi_{j}^{i}(c(t)) \delta q^{j}(t)$. The quasi-infinitesimal variations are scalar functions defined along the curve $c$, therefore their time derivatives $\dot{\zeta}^{i}$ are also defined. The velocity of the system is likewise only defined along the curve $c$, and therefore a 'variation in the velocity' is meaningless without further definition. This was first noted in [2,24], and discussed in [1,3]. To make this rigorous, let us define the following.

\section{Definition 5}

Given a curve $c:\left[t_{1}, t_{2}\right] \rightarrow Q$ and a variation $\vartheta:[-\varepsilon, \varepsilon] \times\left[t_{1}, t_{2}\right] \rightarrow Q$, a vector field $V:[-\varepsilon, \varepsilon] \times$ $[a, b] \rightarrow T Q$ is an extended velocity field on $\vartheta$ if each of the following conditions hold:

1. $V(0, t)=\dot{c}(t)$, for all $t \in\left[t_{1}, t_{2}\right]$,

2. $V\left(s, t_{1}\right)=V\left(s, t_{2}\right)=0$, for all $s \in[-\varepsilon, \varepsilon]$, and

3. $\pi_{Q}($ image $(V))=$ image $(c) \subset Q$, where $\pi_{Q}: T Q \rightarrow Q$ is the projection operator.

\section{Definition 6}

The infinitesimal variation of the extended velocity field $V$ is a vector field $\delta V:\left[t_{1}, t_{2}\right] \rightarrow T Q$ defined along the curve $c$ so that

$$
\delta V(t)=\left.\frac{\partial V(s, t)}{\partial s}\right|_{s=0}
$$


From now on, we will take the quasi-velocities $u^{i}$ to be the quasi-velocities of the extended velocity field $V$, i.e. $V=u^{i} E_{i}$. The following set of transpositional relations then hold, regardless of the choice of extended velocity field.

\section{Theorem 1 (First transpositional relations)}

Let $c:\left[t_{1}, t_{2}\right] \rightarrow Q$ be a curve, $\vartheta:[-\varepsilon, \varepsilon] \times\left[t_{1}, t_{2}\right] \rightarrow Q$ a variation of $c, V:[-\varepsilon, \varepsilon] \times\left[t_{1}, t_{2}\right] \rightarrow T Q$ an extended velocity field on $\vartheta$, and $u^{i}:[-\varepsilon, \varepsilon] \times\left[t_{1}, t_{2}\right] \rightarrow \mathbb{R}$ the quasi-velocities associated with $V$. Then

$$
\left(\frac{d\left(\delta q^{i}\right)}{\mathrm{d} t}-\delta V^{i}\right) \Psi_{i}^{j}=\left(\frac{d\left(\zeta^{j}\right)}{\mathrm{d} t}-\delta u^{j}\right)+\gamma_{a b}^{j} u^{a} \zeta^{b},
$$

where $\gamma_{a b}^{j}$ are the Hamel coefficients

$$
\gamma_{a b}^{j}=\left(\frac{\partial \Psi_{i}^{j}}{\partial q^{k}}-\frac{\partial \Psi_{k}^{j}}{\partial q^{i}}\right) \Phi_{a}^{i} \Phi_{b}^{k} .
$$

For a proof of this theorem, see $[1,2,24]$. A new and more geometric proof that uses the theory of nonholonomic connection is discussed in [5].

The definition of an extended velocity field is an important one, as there are actually an innumerable set of ways in which one can extend the velocity of the curve to the variation [2]. However, two leading ways have dominated the literature. These are referred to as the transitivity choice of Hamel and the transitivity choice of Suslov. For an exposition on the difference between these two choices, see [3,5]. The transitivity choice of Suslov is used both in the context of Suslov's principle [1] as well as the context of a fiber bundle approach to nonholonomic mechanics [25]. When considering the Boltzmann-Hamel equations, however, one uses the transitivity choice of Hamel, which we will use throughout the remainder of this paper:

\section{Definition 7}

Let $c$ be a curve and $\vartheta$ its variation. Then the transitivity choice of Hamel is to define the extended velocity field as the tangents to the varied paths, i.e.

$$
V(s, t)=\frac{\partial \vartheta(s, t)}{\partial t} .
$$

Owing to the continuity of the variation $\vartheta$ it immediately follows that, for the transitivity choice of Hamel, $\delta V=d(\delta c) / \mathrm{d} t$. The transpositional relations then reduce to the equations

$$
\delta u^{j}=\dot{\zeta}^{j}+\gamma_{a b}^{j} u^{a} \zeta^{b} .
$$

When using the transitivity choice of Hamel, one cannot have both $\delta u^{j}=0$ and $\dot{\zeta}^{j}=0$. One must choose between one or the other. The correct dynamical equations of motion are obtained if one chooses the variations so that they satisfy the Lagrange-D'Alembert principle, i.e. so that $\zeta^{\sigma} \equiv 0$. If one, on the other hand, chooses the variations to satisfy $\delta u^{\sigma}=0$, one would obtain trajectories that satisfy Hamilton's principle. Such trajectories are referred to as the vakonomic motion of the system (motion of the variational axiomatic kind), a term introduced in [16].

In Section 4 during our discussion of dynamic nonholonomic control problems, we shall further require the use of quasi-accelerations and quasi-jerks, defined as the time derivatives of the quasivelocities and quasi-accelerations, respectively; i.e. $a^{i}=\dot{u}^{i}, J^{i}=\dot{a}^{i}$. One can easily show that, if one chooses an extended velocity field consistent with the transitivity choice of Hamel, it follows.

\section{Theorem 2 (Second transpositional relation)}

Given a curve $c$, a variation $\vartheta$, and an extended velocity field $V$ that is consistent with the transitivity choice of Hamel (Definition 7), let $u^{i}$ be a set of quasi-velocities of $V$ with respect to some basis $\mathscr{E}$. Then $\delta\left(d u^{i} / \mathrm{d} t\right)=d\left(\delta u^{i}\right) / \mathrm{d} t$. Equivalently, $\delta a^{i}=d\left(\delta u^{i}\right) / \mathrm{d} t$. 
In the next two sections we will show how quasi-velocity techniques may be applied to kinematic and dynamic optimal control problems. Kinematic problems are ones in which one has direct control over an admissible set of velocities. In dynamic problems, one has control over a set of generalized control forces which act on the system.

\section{KINEMATIC OPTIMAL CONTROL}

In this section, we present a quasi-velocity-based method for kinematic optimal control problems, where one has direct controls over the velocities. We begin by defining constrained affine kinematic control systems (e.g. $[15,26,27])$.

\section{Definition 8}

A constrained affine kinematic control system is a triple $(Q, \mathscr{D}, \mathscr{X})$, where $Q$ is a configuration manifold, $\mathscr{D}$ is a smooth, $(n-m)$-dimensional, nonintegrable distribution, and $\mathscr{X}=\left\{X_{I}\right\}_{I=1}^{n-m}$ is a set of $(n-m)$ independent and kinematically admissible vector fields, called the control vector fields, that span the distribution $\mathscr{D}$.

Given a constrained affine kinematic control system, the controls are a set of $(n-m)$ scalar functions $w^{I}:\left[t_{1}, t_{2}\right] \rightarrow \mathbb{R}(I=1, \ldots, n-m)$. Given a set of controls and an initial condition $q_{0} \in Q$, the resultant trajectory is a curve $c:\left[t_{1}, t_{2}\right] \rightarrow Q$ that satisfies the differential equations

$$
\dot{q}^{i}(t)=X_{I}^{i}(q(t)) w^{I}(t), \quad q^{i}(0)=q_{0}^{i},
$$

where $X_{I}^{i}$ is the $i$ th component of the $I$ th control vector field.

\section{Definition 9}

Given a constrained affine kinematic control system $(Q, \mathscr{D}, \mathscr{X})$, two fixed endpoints $q_{1}, q_{2} \in Q$, and a cost functional

$$
I[c]=\int_{t_{1}}^{t_{2}} g(c(t), w(t)) \mathrm{d} t
$$

where $g: Q \times \mathbb{R}^{n-m} \rightarrow \mathbb{R}$ is a smooth function, then the associated kinematic optimal control problem is to determine the control functions $w^{I}:\left[t_{1}, t_{2}\right] \rightarrow \mathbb{R}$ such that the resultant trajectory $c$, i.e. the solution of (5), and the controls $w$, minimize the functional (6) among all kinematically admissible curves.

The idea is to choose the controls as the remaining $n-m$ quasi-velocities. In general, it is always possible to invert the system (5) to solve for the controls as a function of the coordinates and velocities. Though this inversion is not unique, it is typically motivated by the physical system. One can define an $(n-m) \times n$ matrix $b_{i}^{I}$ such that $b_{i}^{I} X_{J}^{i}$ is the $(n-m) \times(n-m)$ identity matrix. Define functions $\varpi^{I}: T Q \rightarrow \mathbb{R}$ such that

$$
\varpi^{I}(q, \dot{q})=b_{i}^{I}(q) \dot{q}^{i} .
$$

The significance of these functions is as follows. Suppose the curve $c=q^{i}(t)$ is a solution of the Equation (5). Then the controls which generate that curve are given by

$$
w^{I}(t)=\varpi^{I}(q(t), \dot{q}(t))=b_{i}^{I}(q(t)) \dot{q}^{i}(t) .
$$

The $\varpi^{I}$, when evaluated along a kinematically admissible curve, are literally the controls which generate that curve. They also constitute a set of $n-m$ quasi-velocities that are linearly independent from the quasi-velocities which arise due to the constraints. We take as quasi-velocities the following set:

$$
\begin{aligned}
& u^{I}=b_{i}^{I}(q) \dot{q}^{i}=\varpi^{I}(q, \dot{q}), \\
& u^{\sigma}=a_{i}^{\sigma}(q) \dot{q}^{i}=0 .
\end{aligned}
$$


Notice that the matrix $\Psi$ will consist of the matrix $b_{i}^{I}$ stacked on top of the matrix $a_{i}^{\sigma}$, and that the first $n-m$ columns of $\Phi$ will coincide with the control vector fields.

We may now rewrite the integrand of the cost function (6) in terms of the quasi-velocities as

$$
C\left(q, u^{I}\right)=g\left(q, \varpi^{I}\right) .
$$

Notice that $C\left(q, u^{I}\right)$ only depends on the $(n-m)$ unconstrained quasi-velocities $u^{I}=\varpi^{I}$.

We wish to minimize the cost functional (6) out of the class of kinematically admissible curves. To do so we add Lagrange multipliers to enforce the nonholonomic constraints before taking the variations. Consider now the functional

$$
\tilde{I}[c]=\int_{t_{1}}^{t_{2}}\left(C(q, u)+\mu_{\sigma} u^{\sigma}\right) \mathrm{d} t .
$$

The addition of the multipliers enforces the constraints and the variations can now be taken to be independent. Taking the variation of this function yields

$$
\delta I=\int_{t_{1}}^{t_{2}}\left(\frac{\partial C}{\partial q^{s}} \Phi_{i}^{s} \zeta^{i}+\frac{\partial C}{\partial u^{I}} \delta u^{I}+\mu_{\sigma} \delta u^{\sigma}\right) \mathrm{d} t
$$

Using the transpositional relations (4) and integrating by parts yields

$$
\delta I=\int_{t_{1}}^{t_{2}}\left[\left(\frac{\partial C}{\partial q^{s}} \Phi_{i}^{s}+\frac{\partial C}{\partial u^{I}} \gamma_{s i}^{I} u^{s}+\mu_{\sigma} \gamma_{s i}^{\sigma} u^{s}\right) \zeta^{i}-\frac{d}{\mathrm{~d} t} \frac{\partial C}{\partial u^{I}} \zeta^{I}-\dot{\mu}_{\sigma} \zeta^{\sigma}\right] \mathrm{d} t .
$$

The variations $\zeta^{i}$ are now independent. Since the solution of the optimal control problem is obtained by setting the variation $\delta I=0$, one obtains a set of differential equations for the optimal path by equating the coefficients of $\zeta^{i}$ to zero. We have therefore proved:

\section{Theorem 3}

Let $\left(Q,\left\{\omega^{\sigma}\right\},\left\{X_{I}\right\}\right)$ and the cost functional $I[c]$ defined in (6) be a kinematic optimal control problem. Let $b^{I}$ be a set of one-forms dual to the control vector fields, so that $\varpi^{I}(q, \dot{q})=b_{i}^{I}(q) \dot{q}^{i}$. Let $a_{i}^{\sigma}$ be the coefficients of the constraint matrix, so that $\omega^{\sigma}=a_{i}^{\sigma} d q^{i}$ and let $\Psi: Q \rightarrow \mathbb{R}^{n \times n}$ be defined by $\Psi_{i}^{\sigma}=a_{i}^{\sigma}$ and $\Psi_{i}^{I}=b_{i}^{I}$. Let $\Phi(q)=\Psi^{-1}(q), u^{i}=\Psi_{j}^{i} \dot{q}^{j}, C(q, u)=g\left(q^{i}, \Psi_{j}^{I} \dot{q}^{j}\right)$, and let $\gamma_{j k}^{i}$ be the Hamel coefficients defined in (3). Then the Boltzmann-Hamel equations for the kinematic optimal control problem are

$$
\begin{aligned}
\frac{d}{\mathrm{~d} t} \frac{\partial C}{\partial u^{I}}-\frac{\partial C}{\partial q^{j}} \Phi_{I}^{j}-\frac{\partial C}{\partial u^{J}} \gamma_{K I}^{J} u^{K} & =\mu_{\tau} \gamma_{K I}^{\tau} u^{K} \quad \text { for } I=1, \ldots, n-m, \\
-\frac{\partial C}{\partial q^{j}} \Phi_{\sigma}^{j}-\frac{\partial C}{\partial u^{J}} \gamma_{K \sigma}^{J} u^{K} & =-\dot{\mu}_{\sigma}+\mu_{\tau} \gamma_{K \sigma}^{\tau} u^{K} \quad \text { for } \sigma=n-m+1, \ldots, n, \\
\dot{q}^{i} & =\Phi_{K}^{i} u^{K} \quad \text { for } i=1, \ldots, n .
\end{aligned}
$$

The solution to these equations is the resultant trajectory of the optimal control problem, and the controls that produce the optimal trajectory is given by

$$
w^{I}(t)=\varpi^{I}(q(t), \dot{q}(t))=\Psi_{i}^{I}(q(t)) \dot{q}^{i}(t), \quad i=1, \ldots, n .
$$

These represent a minimal set of $2 n$ first-order differential equations: the $n-m$ Equations (10) for the unconstrained $u^{I}$ 's, the $m$ Equations (11) for the multipliers $\mu_{\sigma}$ 's, and $n$ kinematic relations (12) for the $q^{i}$ s.

As an interesting aside, if the cost function integrand $C(q, u)$, when expressed in terms of the quasi-velocities, is identical to the constrained Lagrangian, then these equations produce the vakonomic motion associated with the system. See [17] for additional discussion on the coincidence of the vakonomic motion (Lagrange's problem) and the optimal control problem. 


\section{DYNAMIC OPTIMAL CONTROL}

In this section, we will derive a set of Boltzmann-Hamel equations for the dynamic optimal control problem, which is normally a fourth-order system. We will present a minimal set of $4 n-2 m$ first-order differential equations that produces the optimal control. Examples will be discussed in Section 5 .

Definition 10

A constrained affine dynamic control system is a quadruple $(Q, \mathscr{D}, \mathscr{X}, L)$, where $Q$ is a configuration manifold, $\mathscr{D}$ is a smooth, $(n-m)$-dimensional, nonintegrable distribution, $\mathscr{X}=\left\{X^{I}\right\}_{I=1}^{n-m}$ is a set of $(n-m)$ independent covector fields, called the control covector fields, that span the entire cotangent space $T^{*} Q$ when concatenated with the constraint one-forms, and $L$ is a Lagrangian.

Given a constrained affine kinematic control system, the controls are a set of $(n-m)$ scalar functions $w_{I}:\left[t_{1}, t_{2}\right] \rightarrow \mathbb{R}$. Given a set of controls and an initial condition $\left(q_{1}, \dot{q}_{1}\right) \in T Q$, the resultant trajectory is a curve $c:\left[t_{1}, t_{2}\right] \rightarrow Q$ that satisfies the second-order differential equations

$$
\frac{\mathrm{d}}{\mathrm{d} t} \frac{\partial L}{\partial \dot{q}^{i}}-\frac{\partial L}{\partial q^{i}}=\lambda_{\sigma} a_{i}^{\sigma}+X_{i}^{I}(q(t)) w_{I}(t), \quad \dot{q} \in \mathscr{D}, \quad q^{i}\left(t_{1}\right)=q_{1}^{i}, \quad \dot{q}^{i}\left(t_{1}\right)=\dot{q}_{1}^{i},
$$

where $X_{i}^{I}$ is the $i$ th component of the $I$ th control covector field.

\section{Definition 11}

Given a constrained affine dynamic control system $(Q, \mathscr{D}, \mathscr{X}, L)$, two fixed endpoints $\left(q_{1}, \dot{q}_{1}\right)$, $\left(q_{2}, \dot{q}_{2}\right) \in T Q$, and a cost functional

$$
I[c]=\int_{t_{1}}^{t_{2}} g(c(t), \dot{c}(t), w(t)) \mathrm{d} t,
$$

where $g: T Q \times \mathbb{R}^{n-m} \rightarrow \mathbb{R}$ is a smooth function, then the associated dynamic optimal control problem is to determine the control functions $w_{I}:\left[t_{1}, t_{2}\right] \rightarrow \mathbb{R}$ such that the resultant trajectory $c$, i.e. the solution of (13), and the controls $w$, minimize the functional (14) among all kinematically admissible curves.

In order to cast this problem in terms of quasi-velocities, we should like to use the classical Boltzmann-Hamel equations, given below, in lieu of (13).

Theorem 4 (The Boltzmann-Hamel equations)

Let $\mathscr{L}(q, u)=L(q, \dot{q}(q, u))$ be the unconstrained Lagrangian of an $n$ degree of freedom mechanical system subject to $m$ nonholonomic constraints, re-expressed in terms of the quasi-velocities $u$. Suppose the system is also subjected to the applied force $F_{j} d q^{j}$, and let $W_{i}=\Phi_{i}^{j} F_{j}$ be the components of the applied force expressed with respect to the basis $\left\{E_{i}\right\}$. Then the curve of motion satisfies the differential equations

$$
\begin{aligned}
\frac{\mathrm{d}}{\mathrm{d} t} \frac{\partial \mathscr{L}}{\partial u^{I}}-\frac{\partial \mathscr{L}}{\partial q^{j}} \Phi_{I}^{j}-\frac{\partial \mathscr{L}}{\partial u^{j}} \gamma_{J I}^{j} u^{J} & =W_{I} \quad \text { for } I=1, \ldots, n-m, \\
\dot{q}^{i} & =\Phi_{J}^{i} u^{J} \quad \text { for } i=1, \ldots, n .
\end{aligned}
$$

For a proof of these equations, we refer the reader to [1,2]. Notice that Equations (13) are a set of $n$ second-order differential equations coupled with $m$ constraint equations, whereas the Boltzmann-Hamel equations (15)-(16) are a set of $(2 n-m)$ first-order differential equations in which the constraints are enforced trivially by setting $u^{\sigma}=0$. The quasi-velocities should then be taken so that the first $(n-m)$ rows of the matrix $\Psi$ coincide with the control covector fields:

$$
\begin{aligned}
& u^{I}=\Psi_{i}^{I} \dot{q}^{i}=X_{i}^{I} \dot{q}^{i}, \\
& u^{\sigma}=\Psi_{i}^{\sigma} \dot{q}^{i}=a_{i}^{\sigma} \dot{q}^{i}=0 .
\end{aligned}
$$


With this choice, the control forces are $F_{j}=\Psi_{j}^{I} w_{I}$ and the forces with respect to the basis $\left\{E_{i}\right\}$ are simply $W_{I}=\Phi_{I}^{j} \Psi_{j}^{J} w_{J}=w_{I}$ and $W_{\sigma}=0$. Therefore, the controls are precisely identified with the $n-m$ time-varying force functions $W_{I}(t)$ on the right-hand side of (15).

Utilizing (15) and (16), we can rewrite the integrand of the cost function as an explicit function of the coordinates, quasi-velocities, and quasi-accelerations $C(q, u, a)=g(q, \dot{q}(q, u), W(q, u, a))$. Since the Boltzmann-Hamel equations no longer depend on the constrained quasi-velocities and quasi-accelerations, $C(q, u, a)$ is also independent of $u^{\sigma}$ and $a^{\sigma}$. Taking variations yields

$$
\delta I=\int_{t_{1}}^{t_{2}}\left(\frac{\partial C}{\partial q^{i}} \delta q^{i}+\frac{\partial C}{\partial u^{J}} \delta u^{J}+\frac{\partial C}{\partial a^{J}} \delta a^{J}\right) \mathrm{d} t .
$$

Recall that the summation index $J$ runs only over the unconstrained dimensions, $J=1, \ldots, n-m$. Using the second transpositional relations Theorem 2 for $\delta a^{J}$ and then integrating by parts we obtain

$$
\delta I=\int_{t_{1}}^{t_{2}}\left[\frac{\partial C}{\partial q^{i}} \delta q^{i}+\left(\frac{\partial C}{\partial u^{J}}-\frac{d}{\mathrm{~d} t} \frac{\partial C}{\partial a^{J}}\right) \delta u^{J}\right] \mathrm{d} t .
$$

Defining the parameters

$$
\kappa_{J}=\frac{\partial C}{\partial u^{J}}-\frac{d}{\mathrm{~d} t} \frac{\partial C}{\partial a^{J}}
$$

and using the first Transpositional relations (4) we obtain

$$
\delta I=\int_{t_{1}}^{t_{2}}\left[\left(\frac{\partial C}{\partial q^{j}} \Phi_{k}^{j}+\kappa_{J} \gamma_{j k}^{J} u^{j}\right) \zeta^{k}-\dot{\kappa}_{J} \zeta^{J}\right] \mathrm{d} t .
$$

These variations are not free, but subject to the nonholonomic constraints $a_{i}^{\sigma} \dot{q}^{i}=0$. We form the augmented cost integrand by replacing $C(q, u, a)$ with $C(q, u, a)+\mu_{\sigma} u^{\sigma}$. Taking variations, the $\delta \mu^{\sigma}$ coefficients recover the constraints. Ignoring these terms, we are left with

$$
\delta I=\int_{t_{1}}^{t_{2}}\left[\left(\frac{\partial C}{\partial q^{j}} \Phi_{k}^{j}+\kappa_{J} \gamma_{j k}^{J} u^{j}+\mu_{\sigma} \gamma_{j k}^{\sigma} u^{j}\right) \zeta^{k}-\dot{\kappa}_{J} \zeta^{J}-\dot{\mu}_{\sigma} \zeta^{\sigma}\right] \mathrm{d} t,
$$

where the variations are now taken to be unconstrained. Notice the multipliers $\mu_{\sigma}$ are not the mechanical multipliers, but a multiplier on the cost function that enforces Hamilton's principle. We thus have the following:

\section{Theorem 5}

The solution curve of the dynamic optimal control problem (Definition 11) is generated by the system of differential equation:

$$
\begin{aligned}
-\frac{\partial C}{\partial q^{j}} \Phi_{I}^{j}+\dot{\kappa}_{I}-\kappa_{J} \gamma_{K I}^{J} u^{K} & =\mu_{\tau} \gamma_{J I}^{\tau} u^{J} \quad \text { for } I=1, \ldots, n-m ; \\
-\frac{\partial C}{\partial q^{j}} \Phi_{\sigma}^{j}-\kappa_{J} \gamma_{K \sigma}^{J} u^{K} & =\mu_{\tau} \gamma_{J \sigma}^{\tau} u^{J}-\dot{\mu}_{\sigma} \quad \text { for } \sigma=n-m+1, \ldots, n, \\
\dot{q}^{i} & =\Phi_{J}^{i} u^{J} \quad \text { for } i=1, \ldots, n .
\end{aligned}
$$

These equations are the Boltzmann-Hamel equations for the dynamic optimal control problem. The generalized control forces $F_{i}$ are then given by evaluating $W(q, u, a)$ given by the BoltzmannHamel equations (15) and using the transformation relation $F_{i}=\Psi_{i}^{j} W_{j}$.

The optimal control system can therefore be given by a minimal set of $4 n-2 m$ first-order differential equations as follows. We have $n$ kinematic relations (22), $2 n-2 m$ relations $\dot{u}^{I}=a^{I}$ and $\dot{a}^{I}=J^{I}, n-m$ equations for $j^{I}$ (20), and, finally, $m$ relations for the multipliers $\dot{\mu}_{\sigma}(21)$. Once the 


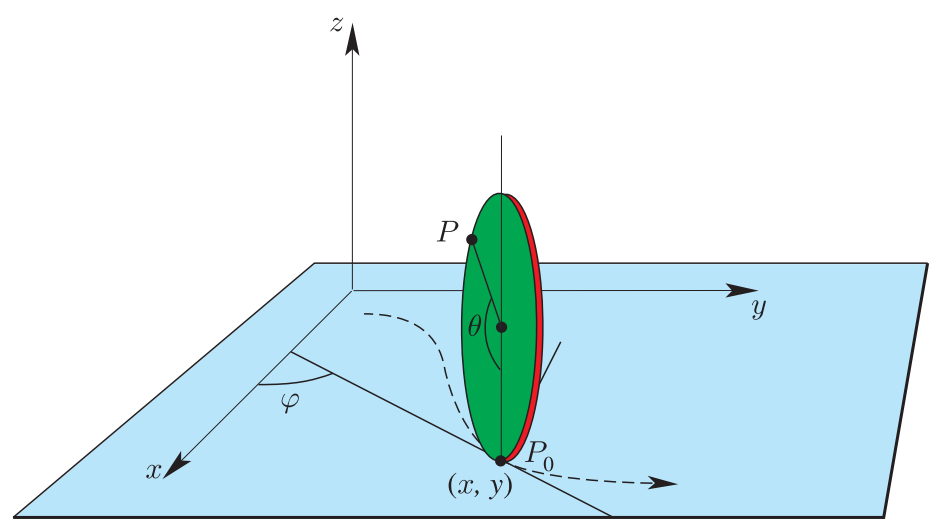

Figure 1. Geometry of the vertical rolling disc.

resulting optimal control dynamics are determined, the control forces which produce the optimal trajectory are then given by the $n-m$ algebraic equations (15). The solution is then found by solving the related boundary value problem, with $4 n-2 m$ prescribed boundary conditions: $q^{i}(0)$, $u^{I}(0), q^{i}(T), u^{I}(T)$.

\section{EXAMPLES}

In this section, we will apply our approach to the kinematic and dynamic vertical rolling disc as well as the dynamic optimal reorientation of a free rigid body. For simplicity, we will restrict our attention to cost functions that minimize the control effort. The procedure, however, can be extended to more general cost functions, though one will not obtain such a clean result.

\subsection{Kinematic optimal control of the vertical rolling disc}

The vertical rolling disc is a basic example of a nonholonomic control system. It is similar in nature to the car-like robot (see [15]), however, the equations work out more cleanly. The generalized coordinates are given by $q=\langle x, y, \theta, \phi\rangle$, where $(x, y)$ is the contact point of the disc on the $x-y$ plane, $\theta$ is the angle that a reference point on the disc makes with the vertical, and $\phi$ is the angle the disc makes with the $x$-axis, see Figure 1 . The motion is subject to the nonholonomic constraints $\dot{x}-\cos (\phi) \dot{\theta}=0$ and $\dot{y}-\sin (\phi) \dot{\theta}=0$. The control velocity field is given by $\dot{q}=X_{1} w^{1}+X_{2} w^{2}$, where $X_{1}=\langle\cos \phi, \sin \phi, 1,0\rangle^{\mathrm{T}}$ and $X_{2}=\langle 0,0,0,1\rangle^{\mathrm{T}}$. One might conceivably have direct control over the angular rates, so that the control functions are given by $\varpi^{1}=\dot{\theta}$ and $\varpi^{2}=\dot{\phi}$. The quasi-velocities are therefore given by $u_{1}=\dot{\theta}, u_{2}=\dot{\phi}, u_{3}=\dot{x}-\cos (\phi) \dot{\theta}$, and $u_{4}=\dot{y}-\sin (\phi) \dot{\theta}$, so that the transformation matrices $\Psi$ and $\Phi$ are given by:

$$
\Psi=\left[\begin{array}{cccc}
0 & 0 & 1 & 0 \\
0 & 0 & 0 & 1 \\
1 & 0 & -\cos \phi & 0 \\
0 & 1 & -\sin \phi & 0
\end{array}\right], \quad \Phi=\left[\begin{array}{cccc}
\cos \phi & 0 & 1 & 0 \\
\sin \phi & 0 & 0 & 1 \\
1 & 0 & 0 & 0 \\
0 & 1 & 0 & 0
\end{array}\right] .
$$

Notice that the top two rows of $\Psi$ correspond to the $b_{i}^{I}$ matrix, which reveal the control functions $\varpi^{I}=b_{i}^{I} \dot{q}^{i}$, whereas the final two rows of $\Psi$ coincide with the constraint matrix $a_{i}^{\sigma}$. The first two columns of the inverse matrix $\Phi$ are the control vector fields; hence $\dot{q}^{i}=\Phi_{j}^{i} u^{j}=\Phi_{I}^{i} u^{I}=X_{I}^{i} w^{I}$. Finally, we confirm that $b_{i}^{I} X_{J}^{i}$ is the $2 \times 2$ identity matrix.

We now consider the following optimal control problem. We wish to determine controls which steer the disc, starting from $q_{1}=q\left(t_{1}\right)$ and stopping at $q_{2}=q\left(t_{2}\right)$, along the path that minimizes 
the cost function: $\frac{1}{2} \int_{t_{1}}^{t_{2}}\left(w_{1}^{2}+w_{2}^{2}\right) \mathrm{d} t$. The nonzero Hamel coefficients are $\gamma_{12}^{3}=\sin \phi=-\gamma_{21}^{3}$ and $\gamma_{21}^{4}=\cos \phi=-\gamma_{12}^{4}$. In terms of the quasi-velocities, the integrand of the cost function becomes $C(q, u)=\frac{1}{2} u_{1}^{2}+\frac{1}{2} u_{2}^{2}$. The Boltzmann-Hamel equations (10)-(12) then produce the following set of first-order differential equations:

$$
\begin{array}{llll}
\dot{u}_{1}=\left(\mu_{4} \cos \phi-\mu_{3} \sin \phi\right) u_{2}, & \dot{\mu}_{3}=0, & \dot{x}=\cos (\phi) u_{1}, & \dot{\theta}=u_{1}, \\
\dot{u}_{2}=\left(\mu_{3} \sin \phi-\mu_{4} \cos \phi\right) u_{1}, & \dot{\mu}_{4}=0, & \dot{y}=\sin (\phi) u_{1}, & \dot{\phi}=u_{2} .
\end{array}
$$

These equations are further discussed in [14]. The solution to this system of differential equations yields the optimal dynamic control equations of the vertical rolling disc. It is equivalent to the following reduced system:

$$
\begin{array}{ll}
\dot{x}=\cos (\phi) \dot{\theta}, & \ddot{\theta}=\left(\mu_{4} \cos \phi-\mu_{3} \sin \phi\right) \dot{\phi}, \\
\dot{y}=\sin (\phi) \dot{\theta}, & \ddot{\phi}=\left(\mu_{3} \sin \phi-\mu_{4} \cos \phi\right) \dot{\theta},
\end{array}
$$

where $\mu_{3}$ and $\mu_{4}$ are constants.

\subsection{Dynamic optimal control of the vertical rolling disc}

Consider the vertical rolling disc with Lagrangian

$$
L=\frac{1}{2}\left(\dot{x}^{2}+\dot{y}^{2}\right)+\frac{1}{4} \dot{\theta}^{2}+\frac{1}{8} \dot{\phi}^{2}
$$

and control torques in the $\theta$ and $\phi$ directions. The dimensions are normalized such that the radius and mass of the disk are unity. The corresponding dynamical equations of motion (see [14]) are

$$
\frac{3}{2} \ddot{\theta}=w_{1}, \quad \frac{1}{4} \ddot{\phi}=w_{2}, \quad \dot{x}=\dot{\theta} \cos \phi \quad \text { and } \quad \dot{y}=\dot{\theta} \sin \phi .
$$

This is equivalent to a minimal set of six first-order differential equations. Since the control forces are in the $\theta$ and $\phi$ directions, we can take the same quasi-velocities obtained using the matrices (23) used in the kinematic case. Note that the control covector fields are the first two rows of $\Psi$.

We now wish to choose the control forces so as to minimize the cost function $\int_{t_{1}}^{t_{2}} \frac{1}{2}\left(w_{1}^{2}+w_{2}^{2}\right) \mathrm{d} t$. Solving for the controls in terms of the quasi-accelerations $w_{1}=\frac{3}{2} \ddot{\theta}=\frac{3}{2} a_{3}$ and $w_{2}=\frac{1}{4} \ddot{\phi}=\frac{1}{4} \ddot{a}_{4}$, this is equivalent to minimizing the action $\int_{t_{1}}^{t_{2}}\left(\frac{9}{8} a_{1}^{2}+\frac{1}{32} a_{2}^{2}\right) \mathrm{d} t$ subject to the nonholonomic constraints. Using the dynamic optimal control Boltzmann-Hamel equations (20) and (21), coupled with the dynamical equations of motion above, and eliminating the controls, we have a minimal system of 12 first-order differential equations:

$$
\begin{aligned}
& \dot{x}=\cos \phi u_{1}, \quad j_{1}=\frac{4}{9}\left(\mu_{3} \sin \phi-\mu_{4} \cos \phi\right) u_{2}, \\
& \dot{y}=\sin \phi u_{1}, \quad j_{2}=16\left(-\mu_{3} \sin \phi+\mu_{4} \cos \phi\right) u_{1}, \\
& \dot{\theta}=u_{1}, \quad \dot{u}_{1}=a_{1}, \quad \dot{a}_{1}=J_{1}, \quad \dot{\mu}_{3}=0, \\
& \dot{\phi}=u_{2}, \quad \dot{u}_{2}=a_{2}, \quad \dot{a}_{2}=J_{2}, \quad \dot{\mu}_{4}=0 .
\end{aligned}
$$

By use of quasi-velocities, quasi-accelerations, and quasi-jerks, we have made the following simplifications: $u_{3}=u_{4}=a_{3}=a_{4}=J_{3}=J_{4}=0$, thereby eliminating the necessity of 6 of the 18 first-order differential equations necessary in the standard approach. The solution to this system of differential equations yields the optimal dynamic control equations of the vertical rolling disc. It is equivalent to the following reduced system:

$$
\begin{array}{ll}
\dot{x}=\cos \phi \dot{\theta}, & \dot{y}=\sin \phi \dot{\theta} \\
\dddot{\theta}=\frac{4}{9}\left(\mu_{3} \sin \phi-\mu_{4} \cos \phi\right) \dot{\phi}, & \dddot{\phi}=16\left(-\mu_{3} \sin \phi+\mu_{4} \cos \phi\right) \dot{\theta},
\end{array}
$$

where $\mu_{3}$ and $\mu_{4}$ are constants. 


\subsection{Dynamic optimal control of the free rigid body}

Consider dynamic control of the free rigid body, where the generalized coordinates are given by the Type-I (aircraft) Euler angles $(\psi, \theta, \phi)$. As quasi-velocities, choose the body-fixed components of the angular momentum $u_{1}=\omega_{x}=-\dot{\psi} \sin \theta+\dot{\phi}, u_{2}=\omega_{y}=\dot{\psi} \cos \theta \sin \phi+\dot{\theta} \cos \phi$, and $u_{3}=\omega_{z}=$ $\dot{\psi} \cos \theta \cos \phi-\dot{\theta} \sin \phi$. The transformation matrices are given as:

$$
\Psi=\left[\begin{array}{ccc}
-\sin \theta & 0 & 1 \\
\cos \theta \sin \phi & \cos \phi & 0 \\
\cos \theta \cos \phi & -\sin \phi & 0
\end{array}\right] \text { and } \Phi=\left[\begin{array}{ccc}
0 & \sec \theta \sin \phi & \sec \theta \cos \phi \\
0 & \cos \phi & -\sin \phi \\
1 & \tan \theta \sin \phi & \tan \theta \cos \phi
\end{array}\right] .
$$

The mechanical Lagrangian is given as $\mathscr{L}(q, u)=\frac{1}{2}\left(I_{x x} u_{1}^{2}+I_{y y} u_{2}^{2}+I_{z z} u_{3}^{2}\right)$. The nonzero Hamel coefficients are $\gamma_{23}^{1}=1, \gamma_{13}^{2}=-1, \gamma_{12}^{3}=1, \gamma_{32}^{1}=-1, \gamma_{31}^{2}=1$, and $\gamma_{21}^{3}=-1$. For notational convenience, define $\eta_{32}=I_{z z}-I_{y y}, \eta_{13}=I_{x x}-I_{z z}$, and $\eta_{21}=I_{y y}-I_{x x}$. Then the Boltzmann-Hamel equations (15) produce the Euler equations

$$
I_{x x} \dot{u}_{1}+\eta_{32} u_{2} u_{3}=M_{x}, \quad I_{y y} \dot{u}_{2}+\eta_{13} u_{1} u_{3}=M_{y}, \quad I_{z z} \dot{u}_{3}+\eta_{21} u_{1} u_{2}=M_{z},
$$

where $M_{x}, M_{y}$, and $M_{z}$ are the control torques applied about the body-fixed principal axes. The cost function integrand $\frac{1}{2}\left(M_{x}^{2}+M_{y}^{2}+M_{z}^{2}\right)$, when expressed in terms of quasi-variables, is given by:

$$
\begin{aligned}
C= & \frac{1}{2}\left\{I_{x x}^{2} a_{1}^{2}+I_{y y} a_{2}^{2}+I_{z z} a_{3}^{2}+2 I_{x x} \eta_{32} a_{1} u_{2} u_{3}+2 I_{y y} \eta_{13} u_{1} a_{2} u_{3}\right. \\
& \left.+2 I_{z z} \eta_{21} u_{1} u_{2} a_{3}+\eta_{32}^{2} u_{2}^{2} u_{3}^{2}+\eta_{13}^{2} u_{1}^{2} u_{3}^{2}+\eta_{21}^{2} u_{1}^{2} u_{2}^{2}\right\} .
\end{aligned}
$$

The $\kappa$ 's (19) are given by:

$$
\begin{aligned}
& \kappa_{1}=I_{y y} \eta_{13} a_{2} u_{3}+I_{z z} \eta_{21} u_{2} a_{3}+\eta_{13}^{2} u_{1} u_{3}^{2}+\eta_{21}^{2} u_{1} u_{2}^{2}-I_{x x} J_{1}-I_{x x} \eta_{32} u_{2} a_{3}-I_{x x} \eta_{32} a_{2} u_{3}, \\
& \kappa_{2}=I_{x x} \eta_{32} a_{1} u_{3}+I_{z z} \eta_{21} u_{1} a_{3}+\eta_{32}^{2} u_{2} u_{3}^{2}+\eta_{21}^{2} u_{1}^{2} u_{2}-I_{y y} J_{2}-\eta_{13} I_{y y} u_{1} a_{3}-\eta_{13} I_{y y} a_{1} u_{3}, \\
& \kappa_{3}=I_{x x} \eta_{32} a_{1} u_{2}+I_{y y} \eta_{13} u_{1} a_{2}+\eta_{32}^{2} u_{2}^{2} u_{3}+\eta_{13}^{2} u_{1}^{2} u_{3}-I_{z z} J_{3}-\eta_{21} I_{z z} u_{1} a_{2}-\eta_{21} I_{z z} a_{1} u_{2} .
\end{aligned}
$$

The optimal control Boltzmann-Hamel equations (20) then work out to be

$$
\dot{\boldsymbol{\kappa}}=\boldsymbol{\kappa} \times \boldsymbol{\omega},
$$

where $\boldsymbol{\kappa}=\left\langle\kappa_{1}, \kappa_{2}, \kappa_{3}\right\rangle$ and $\boldsymbol{\omega}$ is the angular velocity. These provide three differential equations for the $j$ 's. Let $\llbracket$ be the moment inertia tensor with respect to the principal axes basis $\hat{\mathbf{e}}_{x}, \hat{\mathbf{e}}_{y}, \hat{\mathbf{e}}_{z}$, so that, in dyadic notation, $\square=I_{x x} \hat{\mathbf{e}}_{x} \hat{\mathbf{e}}_{x}+I_{y y} \hat{\mathbf{e}}_{y} \hat{\mathbf{e}}_{y}+I_{z z} \hat{\mathbf{e}}_{z} \hat{\mathbf{e}}_{z}$. Let $\Pi:=\square \cdot \boldsymbol{\omega}$ be the body axis angular momentum. Then (25)-(27) can alternatively be re-expressed as:

$$
\boldsymbol{\kappa}=\Pi \times \dot{\Pi}+\Pi \times(\omega \times \Pi)-\ddot{\Pi}-\llbracket \cdot\{2 \omega \times \dot{\Pi}+\dot{\omega} \times \Pi+\omega \times(\omega \times \Pi)\} .
$$

Finally, by defining $\lambda(\omega, \dot{\omega})=\boldsymbol{\kappa}+\ddot{\boldsymbol{\Pi}}$, the dynamic optimal control equations for the free rigid body can be expressed as:

$$
\dddot{\Pi}=\dot{\lambda}+\ddot{\Pi} \times \omega-\lambda \times \omega .
$$

In addition, we have the kinematic relations

$$
\begin{aligned}
& \dot{\psi}=\sec \theta \sin \phi u_{2}+\sec \theta \cos \phi u_{3}, \\
& \dot{\theta}=\cos \phi u_{2}-\sin \phi u_{3}, \\
& \dot{\phi}=u_{1}+\tan \theta \sin \phi u_{2}+\tan \theta \cos \phi u_{3},
\end{aligned}
$$

as well as the relations $\dot{u}_{i}=a_{i}, \dot{a}_{i}=J_{i}$. This is a set of 12 first-order differential equations. Once one solves the corresponding boundary value problem (initial, final Euler angles, angular velocities specified), the controls are determined by the algebraic relations (24). 

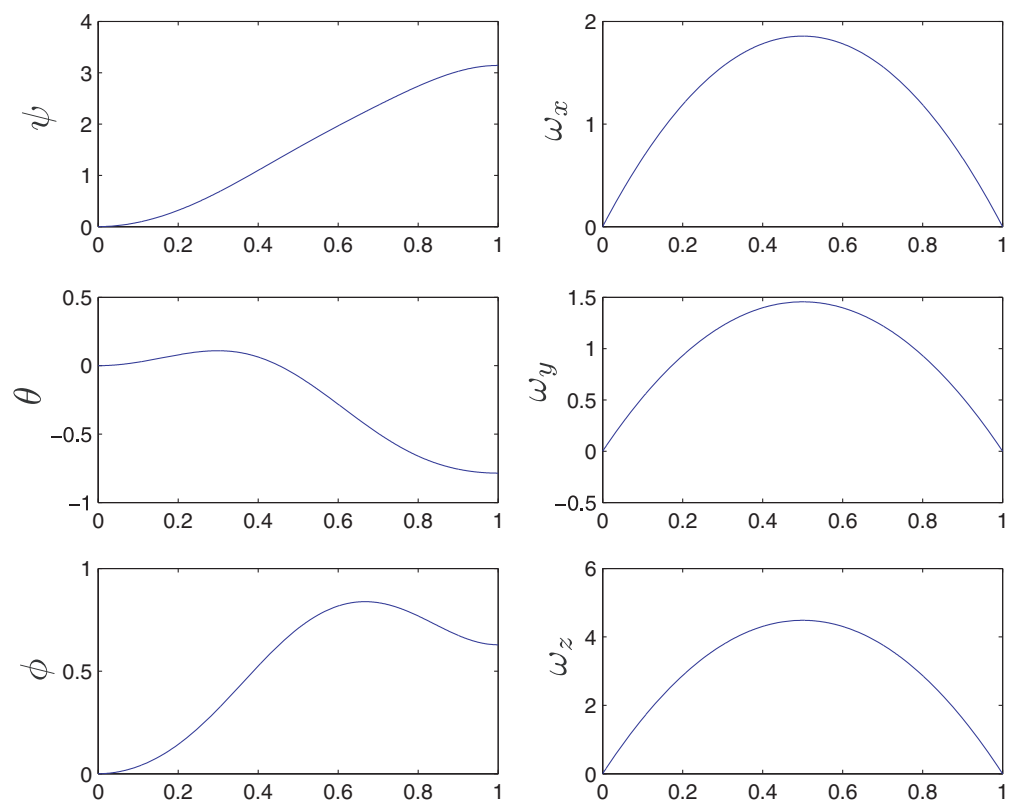

Figure 2. Optimal dynamic control of free sphere: Euler Angles and body-fixed angular velocity with respect to time.

For the special case when the rigid body is spherical one sees from (29) that $\boldsymbol{\kappa}=-\ddot{\boldsymbol{\Pi}}$ and $\boldsymbol{\lambda}=\mathbf{0}$. Then the Boltzmann-Hamel equations for the optimal dynamic control of the free rigid body (30) reduce to $\dddot{\omega}=\ddot{\omega} \times \omega$. When coupled with the kinematic relations (31)-(33) and the algebraic relations (24), the optimal control trajectories of the free rigid sphere are produced. Integrating once yields the second-order system $\ddot{\boldsymbol{\omega}}=\mathbf{c}+\dot{\boldsymbol{\omega}} \times \boldsymbol{\omega}$, which coincides with the result of $[22,28]$. The optimal solution trajectory of the reorientation of the rigid sphere from $\mathbf{q}(0)=\langle 0,0,0\rangle, \omega(0)=$ $\langle 0,0,0\rangle$ to the point $\mathbf{q}(1)=\langle\pi,-\pi / 4, \pi / 5\rangle, \omega(1)=\langle 0,0,0\rangle$ is plotted in Figure 2.

\section{CONCLUSIONS}

In this paper, we showed how one can extend quasi-velocity techniques to kinematic and optimal control problems for mechanical systems with nonholonomic constraints. Standard Lagrange multiplier techniques for kinematical optimal control problems produce a set of $2 n+m$ first-order differential equations: $n$ for the coordinates $q^{i}, n$ for the velocities $\dot{q}^{i}$, and $m$ for the multipliers $\mu_{\sigma}$. On the other hand, by generalizing the dynamic Boltzmann-Hamel equations to the kinematic control setting, we obtain a saving of $m$ first-order differential equations, as one no longer needs to solve the constrained quasi-velocities. Moreover, the differential equations for the multipliers (11) are naturally separated from the differential equations for the quasi-velocities (10).

For the dynamic optimal control problem, one typically encounters a fourth-order system, plus multipliers, which produces a total of $4 n+m$ first-order differential equations, which require further reduction and elimination. The Boltzmann-Hamel form of the equations (Theorem 5) gives a minimal set of $4 n-2 m$ equations of motion, as one no longer need integrate the $m$ constrained quasivelocities, quasi-accelerations, and quasi-jerks, $u^{\sigma} \equiv 0, a^{\sigma} \equiv 0, J^{\sigma} \equiv 0$, respectively. This approach gives us a total saving of $3 m$ first-order differential equations. Initial and final conditions are then enforced by solving the resulting system of differential equations as a two point boundary value problem.

As a final example, we showed that Euler's equations of rigid body dynamics, a special case of the Boltzmann-Hamel equations, can be generalized to a fourth-order form that yields the optimal reorientation trajectory given a set of body-axis control torques. This set of equations could feasibly 
be of some benefit to the optimal reorientation problem of the International Space Station, for example. When one takes the rigid body to be a sphere, these equations reduce to the differential equations defining cubic splines on $S O(3)$.

\section{ACKNOWLEDGEMENTS}

The authors are grateful to the many helpful comments by both reviewers, which helped improve the quality of this paper. The authors further wish to thank support from NSF grants DMS-0604307, DMS-0907949, and CMS-0408542.

\section{REFERENCES}

1. Greenwood DT. Advanced Dynamics. Cambridge University Press: Cambridge, 2003.

2. Neimark JI, Fufaev NA. Dynamics of Nonholonomic Systems. Translations of Mathematical Monographs, vol. 33. AMS: Providence, RI, 1972.

3. Papastavridis JG. Analytical Mechanics. Oxford University Press: Oxford, 2002.

4. Bloch AM, Marsden JE, Zenkov D. Quasi-velocities and symmetries in nonholonomic systems. Preprint, 2009.

5. Maruskin JM, Bloch AM, Marsden JE, Zenkov D. A geometric foundation of quasi-velocities in nonholonomic and variational nonholonomic systems. Preprint, 2009.

6. Greenwood DT. Classical Dynamics. Dover: New York, 1997.

7. Bloch AM, Krishnaprasad PS, Marsden JE, Ratiu TS. The Euler-Poincaré equations and double bracket dissipation. Communications in Mathematical Physics 1996; 175(2):1-42.

8. Marsden JE, Ratiu TS. Introduction to Mechanics with Symmetry. Springer: Berlin, 1994.

9. Maruskin JM. On the dynamical propagation of subvolumes and on the geometry and variational principles of nonholonomic systems. Ph.D. Thesis, The University of Michigan, U.S.A., 2008.

10. Cortes F, Martinez S, Ostrowski JP, McIsaac KA. Optimal gaits for dynamic robotic locomotion. The International Journal of Robotics Research 2001; 20(9):707-728.

11. Koon WS, Marsden JE. Optimal control for holonomic and nonholonomic mechanical systems with symmetry and Lagrangian reduction. SIAM Journal on Control and Optimization 1997; 35(3):901-929.

12. Martinez S, Cortes J, de Leon M. Symmetries in vakonomic dynamics: applications to optimal control. Journal of Geometry and Physics 2001; 38:343-365.

13. Agrachev A, Sachkov Y. Control Theory from the Geometric Viewpoint. Springer: Berlin, 2004.

14. Bloch AM. Nonholonomic Mechanics and Control. Springer: Berlin, 2003.

15. Bullo F, Lewis AD. Geometric Control of Mechanical Systems. Springer: Berlin, 2005.

16. Arnold VI, Kozlov VV, Neishtadt AI. Dynamical Systems III: Mathematical Aspects of Classical and Celestial Mechanics. Springer: Berlin, 1988.

17. Bloch AM, Crouch PE. Reduction of Euler Lagrange problems for constrained variational problems and relation with optimal control problems. The Proceedings of the 33rd IEEE Conference on Decision and Control, Lake Buena Vista, FL, 14-16 December 1994; 2584-2590.

18. Bedrossian N, Bhatt S, Lammers M, Nguyen L, Zhang Y. First ever flight demonstration of zero propellant maneuver attitude control concept. Proceedings of the 2007 Guidance, Navigation and Control Conference, Hilton Head, SC, 20-23 August 2007. AIAA: New York, 2007; 1-12. AIAA 2007-6734.

19. Bedrossian N, Bhatt S. Space station zero propellant maneuver flight results compared to eigenaxis. AAS-08-108, AAS/AIAA Space Flight Mechanics Meeting, Galveston, TX, 27-31 January 2008.

20. Bhatt S. Optimal reorientation of spacecraft using only control moment gyroscopes. Master's Thesis, Rice University, U.S.A., 2007.

21. Kang W, Bedrossian N. Pseudospectral optimal control theory makes debut flight, saves NASA $1 \mathrm{~m}$ in under three hours. SIAM News 2007; 40(7).

22. Noakes L, Heinzinger G, Paden B. Cubic splines on curved spaces. IMA Journal of Mathematical Control and Information 1989; 6:465-473.

23. Maruskin JM, Bloch AM. The Boltzmann-Hamel equations for optimal control. The Proceedings of the 46th IEEE Conference on Decision and Control, New Orleans, LA, 12-14 December 2007; 554-559.

24. Neimark JI, Fufaev NA. Transpositional relations in the analytical mechanics of nonholonomic systems. Journal of Applied Mathematics and Mechanics 1960; 24:1541-1548.

25. Bloch AM, Krishnaprasad PS, Marsden JE, Murray RM. Nonholonomic mechanical systems with symmetry. Archive for Rational Mechanics and Analysis 1996; 136:21-99.

26. Nijmeijer H, van der Schaft AJ. Nonlinear Dynamical Control Systems. Springer: Berlin, 1990.

27. Brockett RW. Control theory and singular Riemannian geometry. New Directions in Applied Mathematics. Springer: Berlin, 1981; 11-27.

28. Crouch P, Silva Leite F. The dynamic interpolation problem: on Riemannian manifolds, Lie groups, and symmetric spaces. Journal of Dynamical and Control Systems 1995; 1(2):177-202. 